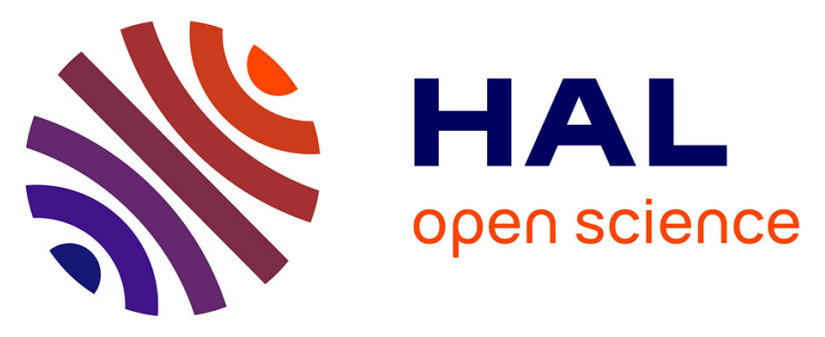

\title{
Two-dimensional hybrid model for magnetic field calculation in electrical machines: exact subdomain technique and magnetic equivalent circuit
}

Brahim Ladghem-Chikouche, Kamel Boughrara, Frédéric Dubas, Rachid Ibtiouen

\section{To cite this version:}

Brahim Ladghem-Chikouche, Kamel Boughrara, Frédéric Dubas, Rachid Ibtiouen. Two-dimensional hybrid model for magnetic field calculation in electrical machines: exact subdomain technique and magnetic equivalent circuit. COMPEL - The international journal for computation and mathematics in electrical and electronic engineering, 2021, 40 (3), pp.535-560. hal-03359943

\section{HAL Id: hal-03359943 https://hal.science/hal-03359943}

Submitted on 30 Sep 2021

HAL is a multi-disciplinary open access archive for the deposit and dissemination of scientific research documents, whether they are published or not. The documents may come from teaching and research institutions in France or abroad, or from public or private research centers.
L'archive ouverte pluridisciplinaire HAL, est destinée au dépôt et à la diffusion de documents scientifiques de niveau recherche, publiés ou non, émanant des établissements d'enseignement et de recherche français ou étrangers, des laboratoires publics ou privés. 


\title{
Two-Dimensional Hybrid Model for Magnetic Field Calculation in Electrical Machines: Exact Subdomain Technique and Magnetic Equivalent Circuit
}

\author{
B. Ladghem-Chikouche, K. Boughrara, F. Dubas, and R. Ibtiouen
}

\begin{abstract}
Purpose - This paper proposes a two-dimensional (2-D) hybrid analytical model (HAM) in polar coordinates, combining a 2-D exact subdomain (SD) technique and magnetic equivalent circuit (MEC), for magnetic field calculation in electrical machines at no-load and on-load conditions.

Design/methodology/approach - In this paper, the proposed technique is applied to dual-rotor permanent-magnet (PM) synchronous machines. The magnetic field is computed by coupling an exact analytical model (AM), based on the formal resolution of Maxwell's equations applied in subdomain, in regions at unitary relative permeability with a MEC using a nodalmesh formulation (i.e., Kirchhoff's current law) in ferromagnetic regions. The AM and MEC are connected in both directions (i.e., $r$ - and $\theta$-edges) of the (non-)periodicity direction (i.e., in the interface between teeth regions and all its adjacent regions as slots and/or air-gap). To provide accuracy solutions, the current density distribution in slots regions is modelled by using Maxwell's equations instead to MEC and characterized by an equivalent magnetomotive force (MMF) situated in slots, teeth and yoke.
\end{abstract}

Findings - It is found that whatever the iron core relative permeability, the developed HAM gives accurate results for noload and on-load conditions. Finite-element analysis (FEA) demonstrates excellent results of the developed technique.

Originality/value - The main objective of this paper is to make a direct coupling between the AM and MEC in both directions (i.e., $r$ - and $\theta$-edges). The current density distribution is modelled by using Maxwell's equations instead to MEC and characterized by an MMF.

Keywords: Hybrid magnetic model, exact subdomain technique, magnetic equivalent circuit, finite-element analysis.

Paper type Research paper

\section{Introduction}

Calculating the performance of electromagnetic devices with high accuracy and geometric detail is often necessary for industrial, automotive and domestic applications with electrical machines. Consideration of magnetic materials in design models (e.g. the saturation effect), as well as accurate knowledge of the magnetic field, are key issues of electrical machine performance evaluation (Dubas and Espanet, 2009). The magnetic field distribution can be evaluated by several approaches, viz., numerical or (semi-)analytical. The better approach is that which gives, with appropriate assumptions, a high precision with fast computation time. Nonlinear numerical and semi-analytical methods are wildly used on modeling electromagnetic devices. For the numerical methods, despite its high calculation accuracy, the computation time remains the major obstacle for study complication and especially for iterative calculations or optimization procedures. Thus, nonlinear semi-analytical models (MEC, Maxwell-Fourier methods) can replace numeric because these approaches have the advantage to be explicit, more or less accuracy, and fast.

A coupling of FEA and MEC (i.e., reluctance or permeance network) has been proposed by (Philips, 1992; Nedjar et al., 2012; Liu et al., 2017). This has given excellent results regarding to high accuracy and reducing time process. In (Sprangers et al., 2015; Sprangers et al., 2016; Djelloul-Khedda et al., 2016; Djelloul-Khedda et al., 2017; Ramakrishnan et al., 2017; Djelloul-Khedda et al., 2018; Djelloul-Khedda et al., 2019; Zhang et al., 2020), a semi-analytical approach based on multi-layer models using the convolution theorem (i.e. Cauchy's product theorem) has been applied to various electrical machines and has given excellent results, although the accuracy of the method can be low for small relative permeability of iron and nonlinear materials. Roubache et al., 2018a presented a contribution on the 2-D SD technique based on the Dubas' superposition technique (Dubas and Boughrara, 2017a, 2017b) by considering the finite soft-magnetic material permeability in spoke-type PM machines. This allows for any non-periodic subdomain. The subdomain connection is performed directly in both directions. The general solutions of Maxwell's equations are deduced by applying the superposition principle by respecting the boundary conditions (BCs) on the various edges of subdomains. This has also been applied to switched reluctance machines supplied by sinusoidal waveform of current (aka, variable flux reluctance machines) (Ben- 


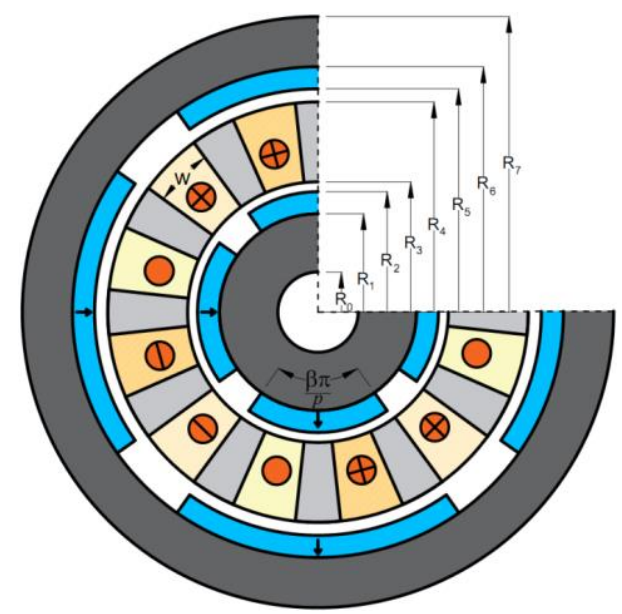

Fig. 1. Dual-rotor PM synchronous machine.

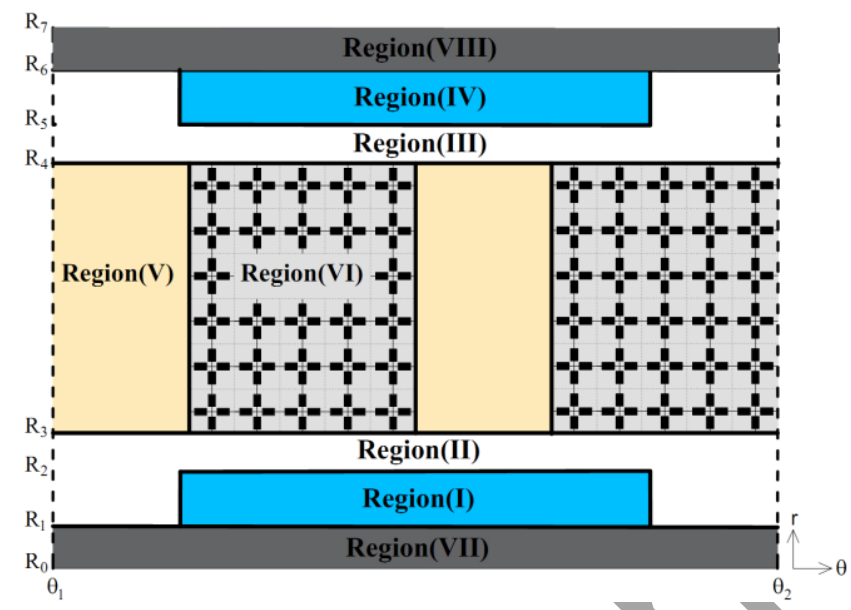

Fig. 2. Longitudinal cross section view of the proposed machine.

Yahia et al., 2018a). Excellent results are obtained whatever the iron relative permeability value, which is constant corresponding to linear zone of the $B(H)$ curve. Nevertheless, the saturation effect in subdomains is global without considering $B(H)$ curve. In (Roubache et al., 2019, 2018b), the authors have extended the proposed model in (Dubas and Boughrara, 2017b) and (Roubache et al., 2018a) to elementary subdomains (E-SDs) in the rotor and/or stator regions with(out) electrical conductivities for full prediction of magnetic field in rotating electrical machines with the local saturation effect solving by the Newton-Raphson iterative algorithm. The introduction of the global or local saturation effect into the semi-analytical calculation requires discretizing all magnetic core materials and uses in each case of discretization a considerable number of harmonics with a view to avoid errors of calculation. Therefore, the exact SD or E-SD technique by inserting ferromagnetic regions is inappropriate for the reduction of computational time. HAM presented by (Ouagued et al., 2015; Laoubi et al., 2015; Ouagued et al., 2016; Bao et al., 2018; Wu et al., 2020; Ceylan et al., 2020) is one of a strong alternative solution to analyze and optimize a large range of electromagnetic devices with the global or local saturation effect. This approach is flexible and gives accurate results under considerable time consuming with respect to numerical methods or formal analytical approach. The aim of this approach is to make a direct coupling between the analytical solution obtained from Maxwell's equations in simple regions (e.g., the air-gap or PM region) and MEC in more complex regions (e.g., slots and teeth regions). Another strong advantage of this approach is its flexibility against stationary and moving parts (Demenko et al., 2008; Ullah et al., 2018). However, in all the papers dealing with HAM, the coupling between the conventional SD technique (without superposition technique) and (non)linear MEC is given only in $\theta$-direction. Moreover, the current density contributions in the magnetic field are represented by magnetomotive forces (MMFs) situated in the yoke, slots and teeth. Those MMFs are sources of inaccuracies in the HAM. Also, the semianalytical model used in the HAM cannot be coupled to the MEC in both directions (i.e., $r$ - and $\theta$-edges).

In this paper, an improved hybrid analytical modeling is proposed and applied to dual-rotor PM synchronous machines (Golovanov et al., 2019a, 2019b). The developed 2-D HAM uses the exact SD technique (Dubas and Boughrara, 2017b) able to be coupled to the MEC, using a nodal-mesh formulation (i.e., Kirchhoff's current law), in both directions (i.e., $r$ - and $\theta$-edges) and permits to avoid the using of MMFs that represents the current densities in slots. From now on, the HAM permits to model all ferromagnetic regions with MEC and all non-magnetic regions (e.g., the air-gap, slots and PMs) with the exact SD technique. The advantage of this HAM is the introduction of local saturation effect which can be done easily.

\section{Model Definition and Assumptions}

The dual-rotor PM synchronous machine, represented in Fig. 1, is divided into eight regions, as shown on Fig. 2, viz.,

1) PMs: Region I and IV; 
2) Vacuum: Region II and III;

3) $Q$ slots with coils: Region $\mathrm{V}$ with the index $x=1 . . Q$;

4) $Q$ soft-material: Region VI with the index $x=1 . . Q$;

5) Iron yoke: Region VII and VIII.

This machine is modelled on a 2-D polar coordinate system. The magnetic field solution is based on the following simplifying assumptions:

1) the problem is quasi-static;

2) the magnetic vector potential in non-magnetic regions (i.e., the air-gap, slots and PMs) has only one component along $z$-axis, i.e., $\boldsymbol{A}=\left\{0 ; 0 ; A_{z}\right\}$;

3) the electrical conductivity in all regions is supposed null;

4) all regions are considered as isotropic;

5) the PMs are considered to have a linear second quadrant characteristic;

6) all regions have radial sides.

\section{Formulation of HAM}

\section{A. Introduction}

In this paper, two approaches are presented:

- Exact AM based on the formal resolution of Maxwell's equations applied in subdomain by applying the separation of variables method, the Fourier's series, and the superposition technique;

- $\quad$ MEC of which mesh elements can be discretized into one or several bidirectional (BD) blocks.

The exact AM is proposed for the regions I, II III, IV and V. Using the magnetostatic Maxwell's equations, the general partial differential equation (PDE) can be expressed in terms of $\boldsymbol{A}$ with $\mu=C^{\text {st }}$ can be expressed by (Dubas and Boughrara, 2017a)

$$
\nabla^{2} \boldsymbol{A}=-\left[\mu \boldsymbol{J}+\mu_{0} \nabla \times \boldsymbol{M r}\right]
$$

where $\boldsymbol{J}$ is the current density (due to supply currents) vector, $\boldsymbol{M r}$ is the magnetization vector (with $\boldsymbol{M r}=$ 0 for the vacuum/iron or $\boldsymbol{M r} \neq 0$ for the PMs according to the magnetization direction), and $\mu=\mu_{0} \cdot \mu_{r}$ is the absolute magnetic permeability of the magnetic material in which $\mu_{0}$ and $\mu_{r}$ are respectively the vacuum permeability and the relative permeability of the magnetic material (with $\mu_{r}=1$ for the vacuum or $\mu_{r} \neq 1$ for the PMs/iron).

The MEC is adopted for the region VI and based on the nodal-mesh formulation (i.e., Kirchhoff's current law) in terms of magnetic scalar potential $\boldsymbol{u}$. The magnetic field intensity vector $H$ can be related to the scalar magnetic potential by

$$
\boldsymbol{H}=-\nabla \boldsymbol{u}
$$

The main objective of this technique is to make a direct coupling between the region VI, which has relative permeability different to unity, and its adjacent regions (viz., Regions II, III and V). For simplicity and to avoid introducing the regions VII and VIII in the system to be solved, the relative permeability of these regions is supposed equal to infinity. It is easy to add these regions in the HAM.

\section{B. Exact SD Technique}

By using (1), the general PDEs in terms of $\boldsymbol{A}$ in various regions can be written as

$$
\begin{aligned}
& \nabla^{2} \boldsymbol{A}=-\mu_{o} \nabla \times \boldsymbol{M r} \quad \text { in Regions I and IV } \\
& \nabla^{2} \boldsymbol{A}=0 \quad \text { in Regions II and III } \\
& \nabla^{2} \boldsymbol{A}=-\mu_{0} \boldsymbol{J} \quad \text { in Region } \mathrm{V}
\end{aligned}
$$




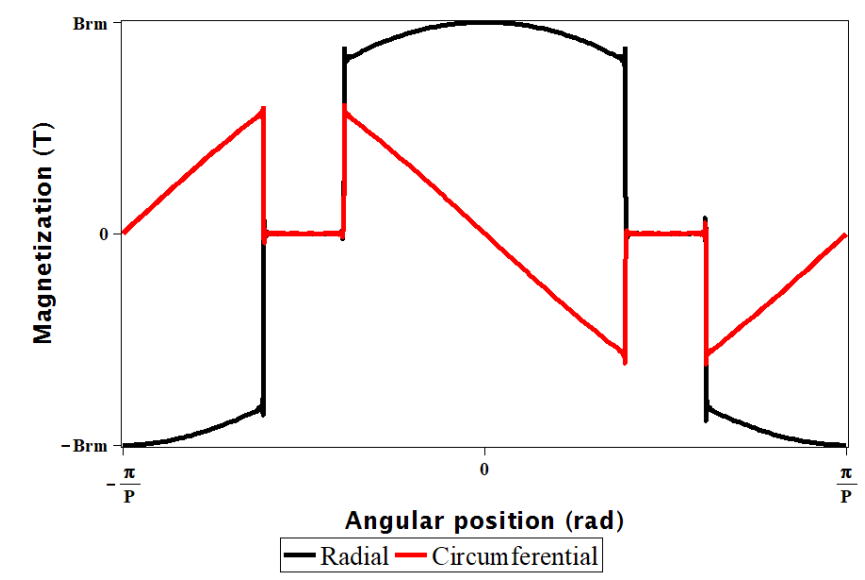

Fig. 3. Parallel magnetization waveforms.

The magnetization vector $\boldsymbol{M r}$ of PMs can be expressed by

$$
\boldsymbol{M r}=M r_{r} \boldsymbol{a}_{\boldsymbol{r}}+M r_{\theta} \boldsymbol{a}_{\boldsymbol{\theta}}
$$

where $M r_{r}$ and $M r_{\theta}$ are respectively the radial and circumferential magnetization. The proposed electrical machine with a parallel magnetization (see Fig. 3) has anti-periodicity equal to $\pi / p$ where $p$ is the number of pole pairs. The components of $\boldsymbol{M r}$ can be specified explicitly by Fourier's series as

$$
\begin{aligned}
& M r_{\theta}=\sum_{n} M r_{\theta s n} \cdot \sin (n p \theta)+M r_{\theta c n} \cdot \cos (n p \theta) \\
& M r_{r}=\sum_{n} M r_{r s n} \cdot \sin (n p \theta)+M r_{r c n} \cdot \cos (n p \theta)
\end{aligned}
$$

where $n$ is the spatial harmonic order. The expressions of $M r_{\theta s n}, M r_{\theta c n}, M r_{r s n}$ and $M r_{r c n}$ are detailed in Appendix A.

The field vectors $\boldsymbol{B}=\left\{B_{r} ; B_{\theta} ; 0\right\}$ and $\boldsymbol{H}=\left\{H_{r} ; H_{\theta} ; 0\right\}$ are coupled by the magnetic material equation

$$
\begin{array}{ll}
\boldsymbol{B}=\mu_{m} \boldsymbol{H}+\mu_{o} \boldsymbol{M r} & \text { with } \mu_{m} \cong \mu_{o} \text { in Regions I and IV } \\
\boldsymbol{B}=\mu_{0} \boldsymbol{H} & \text { in Regions II, III and V }
\end{array}
$$

Using $\boldsymbol{B}=\nabla \times \boldsymbol{A}$, the components of $\boldsymbol{B}$ can be deduced by

$$
B_{r}=\frac{1}{r} \cdot \frac{\partial A_{z}}{\partial \theta} \quad \& \quad B_{\theta}=-\frac{\partial A_{z}}{\partial r}
$$

In polar coordinates $(r, \theta),(3)$ in terms of $\boldsymbol{A}=\left\{0 ; 0 ; A_{z}\right\}$ can be rewritten as:

- in the Region I and IV (i.e., Poisson's equation):

$$
\frac{\partial^{2} A_{z}^{I, I V}}{\partial r^{2}}+\frac{1}{r} \cdot \frac{\partial A_{z}^{I, I V}}{\partial r}+\frac{1}{r^{2}} \cdot \frac{\partial^{2} A_{z}^{I, I V}}{\partial \theta^{2}}=-\frac{\mu_{0}}{r} \cdot\left(M r_{\theta}-\frac{\partial M r_{r}}{\partial \theta}\right)
$$

- $\quad$ in the Region II and III (i.e., Laplace's equation):

$$
\frac{\partial^{2} A_{z}^{I I, I I I}}{\partial r^{2}}+\frac{1}{r} \frac{\partial A_{z}^{I I, I I I}}{\partial r}+\frac{1}{r^{2}} \frac{\partial^{2} A_{z}^{I I, I I I}}{\partial \theta^{2}}=0
$$

- in the Region V (i.e., Poisson's equation):

$$
\frac{\partial^{2} A_{z}^{V}}{\partial r^{2}}+\frac{1}{r} \cdot \frac{\partial A_{z}^{V}}{\partial r}+\frac{1}{r^{2}} \cdot \frac{\partial^{2} A_{z}^{V}}{\partial \theta^{2}}=-\mu_{0} \cdot J_{z}
$$


Based on the separation of variables method and the Fourier series expansion, the general solution of $A_{z}$ can be described as:

- in the Region I and IV:

$$
A_{z}^{I, I V}=\begin{aligned}
& \sum_{n}\left(C_{3 n}^{I, I V} \cdot r^{n p}+C_{4 n}^{I, I V} \cdot r^{-n p}+\Gamma_{S}\right) \cdot \sin (n p \theta)+\cdots \\
& \sum_{n}\left(C_{5 n}^{I, I V} r^{n p}+C_{6 n}^{I, I V} \cdot r^{-n p}+\Gamma_{c}\right) \cdot \cos (n p \theta)
\end{aligned}
$$

where

$$
\begin{gathered}
\Gamma_{s}= \begin{cases}\frac{n p M r_{r s n}-M r_{\theta s n}}{(n p)^{2}-1} \cdot r & \text { if } n p \neq 1 \\
\frac{M r_{r s 1}-M r_{\theta s 1}}{2} \cdot r \cdot \ln (r) & \text { if } n p=1\end{cases} \\
\Gamma_{c}= \begin{cases}-\frac{n p M r_{r c n}-M r_{\theta c n}}{(n p)^{2}-1} \cdot r & \text { if } n p \neq 1 \\
-\frac{M r_{r c 1}-M r_{\theta c 1}}{2} \cdot r \cdot \ln (r) \text { if } n p=1\end{cases}
\end{gathered}
$$

These regions are $2 \pi$ periodic and have interfaces with other regions depending only on the $\theta$-direction.

- $\quad$ in the Region II and III:

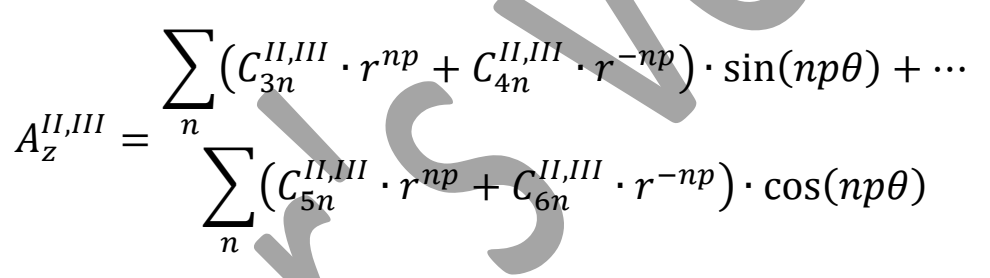

These regions are also $2 \pi$ periodic and have interfaces with other regions depending only on the $\theta$ direction.

- in the Region V:

$$
\begin{gathered}
C_{x 1}^{V}+C_{x 2}^{V} \cdot \ln (r)-\frac{1}{4} \cdot \mu_{o} \cdot J_{z x} \cdot r^{2}+\cdots \\
A_{z x}^{V}=\sum_{m} G_{x m}^{\theta} \cdot \cos \left[\beta_{m} \cdot\left(\theta-g_{x}+\frac{w}{2}\right)\right]+\cdots \\
\sum_{v} G_{x v}^{r} \cdot \sin \left[\lambda_{v} \cdot \ln \left(\frac{r}{R_{3}}\right)\right] \\
G_{x v}^{r}=C_{x 5 v}^{V} \cdot \frac{\sinh \left[\lambda_{v} \cdot\left(\theta-g_{x}+\frac{w}{2}\right)\right]}{\sinh \left(\lambda_{v} \cdot w\right)}+C_{x 6 v}^{V} \cdot \frac{\sinh \left[\lambda_{v} \cdot\left(\theta-g_{x}-\frac{w}{2}\right)\right]}{\sinh \left(\lambda_{v} \cdot w\right)}
\end{gathered}
$$

with

$$
J_{z x}=J_{m} \cdot\left[\begin{array}{llllllllllll}
1 & 1 & 0 & -1 & -1 & 0 & 1 & 1 & 0 & -1 & -1 & 0
\end{array}\right]
$$

where $J_{m}$ is the current density peak, $g_{x}$ is the position of $x^{\text {th }}$ coils, $m$ and $v$ are the spatial harmonic orders, 
$\beta_{m}$ and $\lambda_{v}$ are the spatial frequency (or periodicity) in both directions defined by

$$
\beta_{m}=\frac{m \pi}{w} \quad \& \quad \lambda_{v}=\frac{v \pi}{\ln \left(R_{4} / R_{3}\right)}
$$

As can be shown in (13), the analytical solution is the superposition of two components in $r$ - and $\theta$ directions based on the superposition technique (Dubas and Boughrara, 2017a, 2017b; Roubache et al., 2018a). This solution permits the coupling with the MEC of regions VI in both directions (i.e., $r$ - and $\theta$ edges).

\section{MEC (viz., Reluctance or Permeance Network)}

In region VI, the solution of $\boldsymbol{u}$ can be achieved according to Kirchhoff's laws. According to Fig. 4, each node (i) inside region VI is connected to four neighbouring nodes, except those situated between region II, region III and region V.

For this case, the magnetic flux $\varphi$ can be written as:

$$
\begin{gathered}
\sum_{j=a, b, c, d} \varphi_{x, i j}=0 \\
u_{x, i}-u_{x, j}=\frac{\varphi_{x, i j}}{P_{x, i j}}
\end{gathered}
$$

and then,

$$
\begin{gathered}
u_{x, i} \sum_{j=a, b, c, d} P_{x, i j}-\sum_{j=a, b, c, d} P_{x, i j} \cdot u_{x, j}=0 \\
P_{x, i j}=\frac{1}{\Re_{x, i j}}=\mu_{0} \cdot \mu_{r} \cdot \frac{S_{x, i j}}{L}
\end{gathered}
$$

where $L, S_{x, i j}$ and $\mu_{r}$ are respectively, active length, section and relative permeability of the reluctance element $\Re$.

Using (2), the components of $\boldsymbol{H}$ can be deduced by

$$
H_{r}=-\frac{\partial u}{\partial r} \quad \& \quad H_{\theta}=-\frac{1}{r} \cdot \frac{\partial u}{\partial \theta}
$$

Since (18) are applicable for the analytical solutions of Maxwell's equations. These equations should be

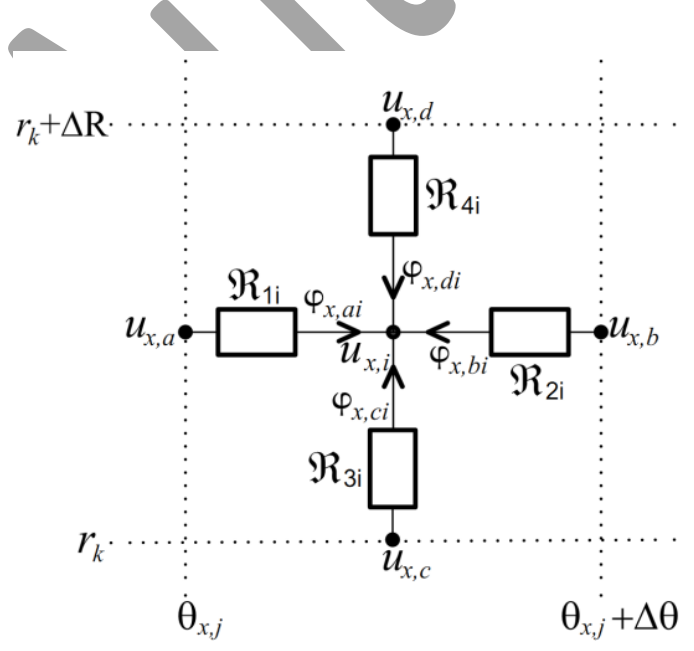

Fig. 4. 2-D Reluctance elements $\Re$, magnetic potential $u$ and magnetic flux $\varphi$.

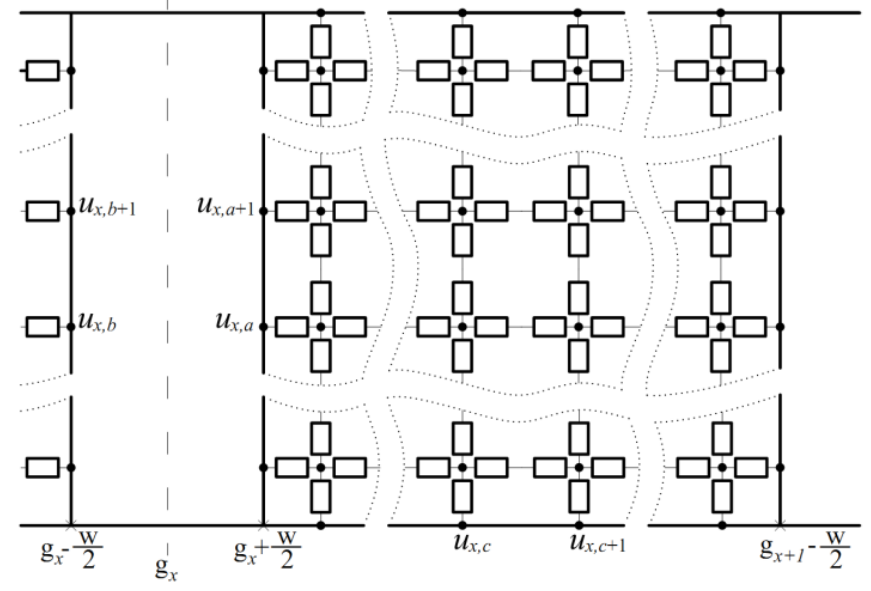

Fig. 5. Uniform mesh of the region VI discretized into several BD blocks. 
rewritten using numerical differentiation defined as the limit of a difference quotient as:

$$
H_{r}(r)=\lim _{\Delta r \rightarrow 0}\left(-\frac{\Delta u}{\Delta r}\right) \& H_{\theta}(\theta)=\lim _{\Delta \theta \rightarrow 0}\left(-\frac{1}{r} \cdot \frac{\Delta u}{\Delta \theta}\right)
$$

The difference quotient $H_{r}(r)$ and $H_{\theta}(\theta)$ is a derivative approximation. This gets better as $\Delta r$ and $\Delta \theta$ gets smaller.

\section{Boundary Conditions and Linear Systems}

To give the final solution of the equations system, the BCs should be defined by equalizing potential vector magnetic, radial component of $\boldsymbol{B}$ and circumferential component of $\boldsymbol{H}$ in the $r$-and $\theta$-direction.

On the $\theta$-direction:

- At $r=R_{1}$ :

$$
H_{\theta}^{I}\left(R_{1}, \theta\right)=0 \quad \forall \theta
$$

- At $r=R_{2}$ :

$$
\begin{array}{ll}
A_{z}^{I}\left(R_{2}, \theta\right)=A_{z}^{I I}\left(R_{2}, \theta\right) & \forall \theta \\
H_{\theta}^{I}\left(R_{2}, \theta\right)=H_{\theta}^{I I}\left(R_{2}, \theta\right) & \forall \theta
\end{array}
$$

- At $r=R_{3}$ :

$$
\begin{gathered}
P_{x, c i}\left(u_{x, c}-u_{x, i}\right)=L R_{3} \cdot \int_{\theta_{x, j}}^{\theta_{x, j}+\Delta \theta} B_{r}^{I I}\left(R_{3}, \theta\right) \cdot d \theta \\
\left.\left(A_{z}^{I I}\left(R_{3}, \theta\right)=A_{z x}^{V}\left(R_{3}, \theta\right)\right)\right|^{g_{x}-\frac{w}{2} \leq \theta \leq g_{x}+\frac{w}{2}} \\
H_{\theta}^{I I}\left(R_{3}, \theta\right)=\sum_{x=1}^{Q}\left(\left.H_{\theta x}^{V}\left(R_{3}, \theta\right)\right|^{g_{x}-\frac{w}{2} \leq \theta \leq g_{x}+\frac{w}{2}}+\left.H_{\theta x}^{V I}\left(R_{3}, \theta\right)\right|^{g_{x}+\frac{w}{2} \leq \theta \leq g_{x+1}-\frac{w}{2}}\right)
\end{gathered}
$$

In order to satisfy (25), the magnetic flux intensity $H_{\theta x}^{V I}(r, \theta)$ by applying (19) should be written as:

$$
H_{\theta x}^{V I}(r, \theta)=-\sum_{c=1}^{N C-1} \sum_{\nu} \frac{1}{r} \cdot\left(\frac{u_{x, c+1}-u_{x, c}}{\Delta \theta}\right) \times\left[h_{\theta x s v}^{V I} \cdot \sin (v p \theta)+h_{\theta x c v}^{V I} \cdot \cos (v p \theta)\right]
$$

where $N C$ is the number of reluctance rows, and $h_{\theta x s v}^{V I} \& h_{\theta x c v}^{V I}$ are the Fourier constants.

- At $r=R_{4}$

$$
\begin{gathered}
P_{x, i d}\left(u_{x, i}-u_{x, d}\right)=L R_{4} \cdot \int_{\theta_{x, j}}^{\theta_{x, j}+\Delta \theta} B_{r}^{I I I}\left(R_{4}, \theta\right) \cdot d \theta \\
\left.\left(A_{z}^{I I I}\left(R_{4}, \theta\right)=A_{z x}^{V}\left(R_{4}, \theta\right)\right)\right|^{g_{x}-\frac{w}{2} \leq \theta \leq g_{x}+\frac{w}{2}} \\
H_{\theta}^{I I I}\left(R_{4}, \theta\right)=\sum_{x=1}^{Q}\left(\left.H_{\theta x}^{V}\left(R_{4}, \theta\right)\right|^{g_{x}-\frac{w}{2} \leq \theta \leq g_{x}+\frac{w}{2}}+\left.H_{\theta x}^{V I}\left(R_{4}, \theta\right)\right|^{g_{x}+\frac{w}{2} \leq \theta \leq g_{x+1}-\frac{w}{2}}\right)
\end{gathered}
$$

- At $r=R_{5}$ :

$$
\begin{array}{ll}
A_{z}^{I I I}\left(R_{5}, \theta\right)=A_{z}^{I V}\left(R_{5}, \theta\right) & \forall \theta \\
H_{\theta}^{I I I}\left(R_{5}, \theta\right)=H_{\theta}^{I V}\left(R_{5}, \theta\right) & \forall \theta
\end{array}
$$




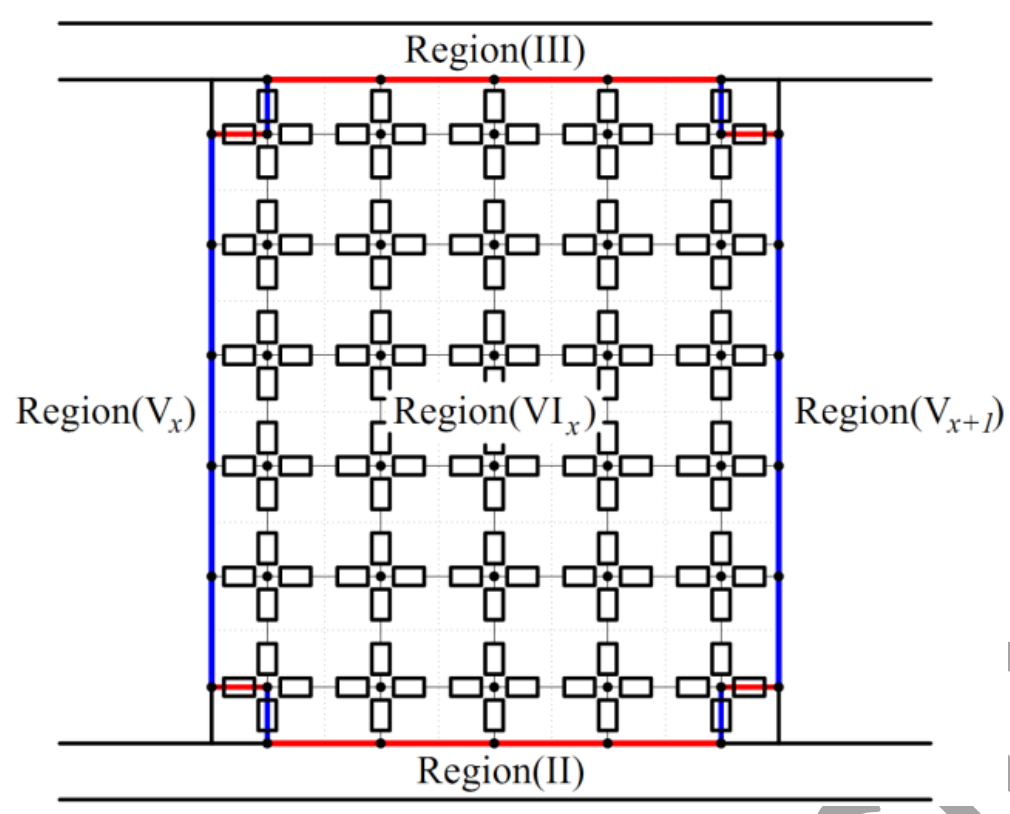

Fig. 6. Proposed BCs between: blue colour region VI and region V, and red colour region VI and regions II \& III.

- At $r=R_{6}$ :

$$
H_{\theta}^{I V}\left(R_{6}, \theta\right)=0 \quad \forall \theta
$$

On the $r$-direction, viz., on the edges of the region $\mathrm{V}$ and the region $\mathrm{VI}$ :

- $\operatorname{for} \theta=g_{x}+w / 2$ :

$$
\begin{gathered}
P_{x, a i}\left(u_{x, a}-u_{x, i}\right)=L \int_{r_{k}}^{r_{k}+\Delta \mathrm{R}} B_{\theta x}^{V}\left(r, g_{x}+\frac{w}{2}\right) \cdot d r \\
H_{r x}^{V}\left(r, g_{x}+\frac{w}{2}\right)=H_{r x}^{V I}(r)
\end{gathered}
$$

In order to satisfy (34), the magnetic flux intensity $H_{r x}^{V I}(r)$ by applying (19) should be written as:

$$
H_{r x}^{V I}(r)=-\sum_{a=1}^{N L-1} \sum_{v}\left(\frac{u_{x, a+1}-u_{x, a}}{\Delta r}\right) \times h_{r x v}^{V I} \cdot \sin \left[\lambda_{v} \cdot \ln \left(\frac{r}{R_{3}}\right)\right]
$$

where $N L$ is the number of reluctance columns, and $h_{r x v}^{V I}$ is the Fourier constant.

- for $\theta=g_{x}-w / 2$.

$$
\begin{gathered}
P_{x-1, i b}\left(u_{x-1, i}-u_{x-1, b}\right)=L \cdot \int_{r_{k}}^{r_{k}+\Delta R} B_{\theta x}^{V}\left(r, g_{x}-\frac{w}{2}\right) \cdot d r \\
H_{r x}^{V}\left(r, g_{x}-\frac{w}{2}\right)=H_{r(x-1)}^{V I}(r)
\end{gathered}
$$

Again, to satisfy (37), the magnetic flux intensity $H_{r x}^{V I}(r)$ in the left side of the region $\mathrm{V}$ should be written as (35) by changing the variable $a$ by $b$ (see Fig. 5).

For simplicity's sake, the proposed machine can be modelled for half of the period. For this case, antiperiodic BCs are proposed at $\theta=\theta_{1}$ and $\theta=\theta_{2}$ :

$$
P_{Q, i b}\left(u_{Q, i}-u_{Q, b}\right)=-L \cdot \int_{r_{k}}^{r_{k}+\Delta R} B_{\theta 1}^{V}\left(r, g_{1}-\frac{w}{2}\right) \cdot d r
$$




$$
H_{r Q}^{V}\left(r, g_{Q}-\frac{w}{2}\right)=-H_{r 1}^{V I}(r)
$$

Fig. 6 shows a regular discretization of the region VI. Since there are no nodes in the corners in this region, a direct coupling between both models is done by respecting the interface indicated by blue and red colour.

It can be seen that all BCs are defined and the correlation of Fourier constants can be found as detailed in Appendix B. Thus, the system of linear algebraic equations can be written as:

$$
[A] \cdot[X]=[b]
$$

where $[A](N \times N)$ is the topological matrix, $[b](N \times 1)$ is the vector contain all flux source parameters and $[X](N \times 1)$ is the vector contain all unknow Fourier constants to be determined, in which $N=$ $12 n h+(2+4 N h+N L(N C+2)+2 N C) Q$ with $N h$ and $n h$ being respectively the number of harmonics in the slot and tooth region and in other regions.

\section{Comparison of Hybrid Analytical and Finite-Element Calculations}

Figs. $7 \sim 10$ present a comparison between HAM and finite elements analysis FEA obtained from FEMM software (Meeker, 2010) for the open-circuit magnetic flux distribution with a parallel magnetization pattern and for the armature reaction magnetic flux distribution in the middle of the region II with different values of iron relative permeability (i.e., region VI). The proposed electrical machine parameters are shown in Table I. These results have been calculated under an acceptable number of discretization of the region VI $(N C=30$ and $N L=15)$. Excellent agreement is achieved between both models.
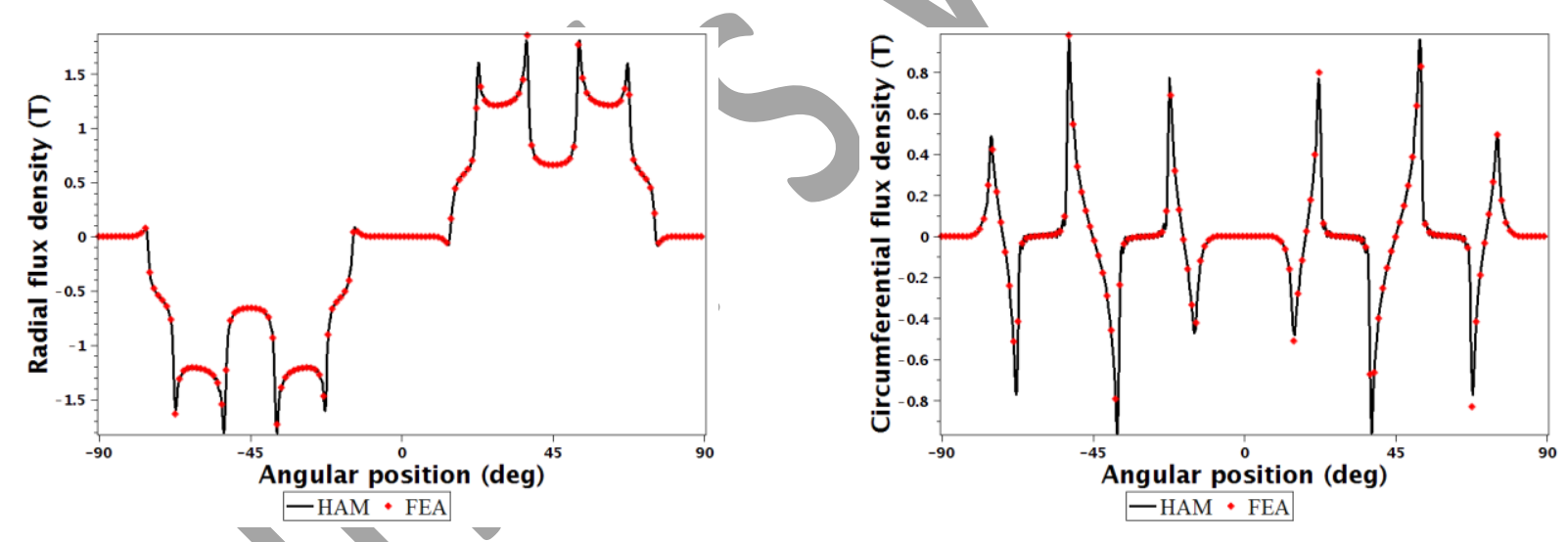

Fig. 7. Comparison of HAM and FEA predicted for the open circuit magnetic flux density distribution with a parallel magnetization pattern in the middle of region II for $\mu_{r}=1,000$ in the region VI.
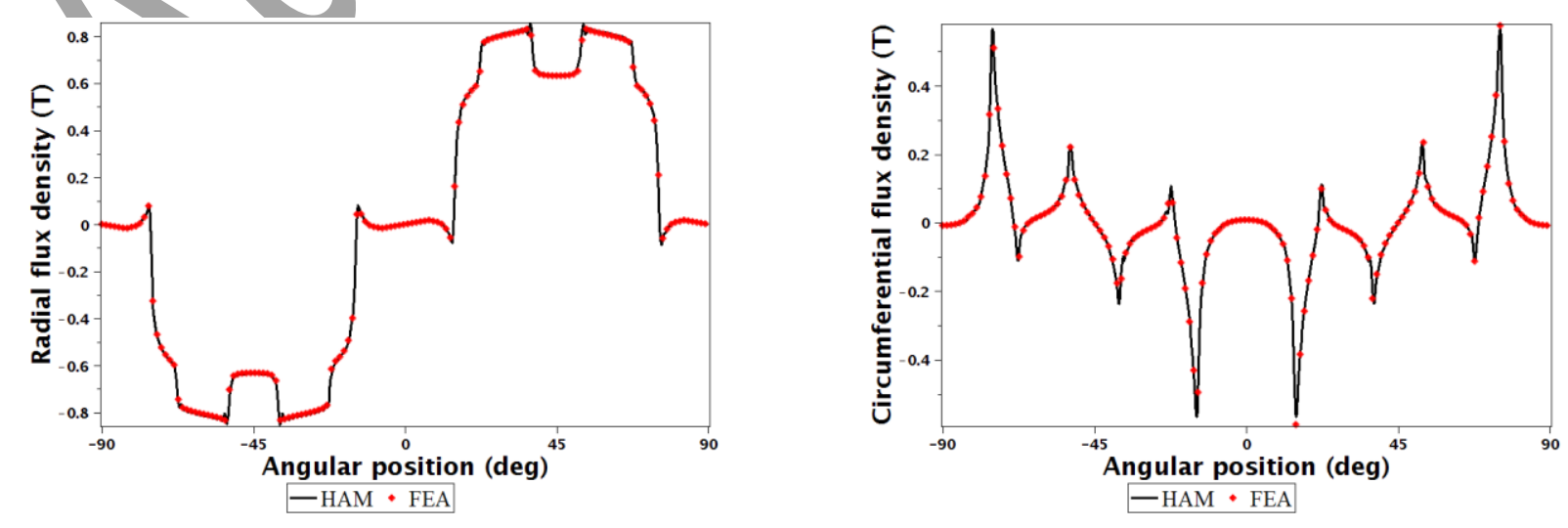

Fig. 8. Comparison of HAM and FEA predicted for the open circuit magnetic flux density distribution with a parallel magnetization pattern in the middle of region II for $\mu_{r}=2$ in the region VI. 

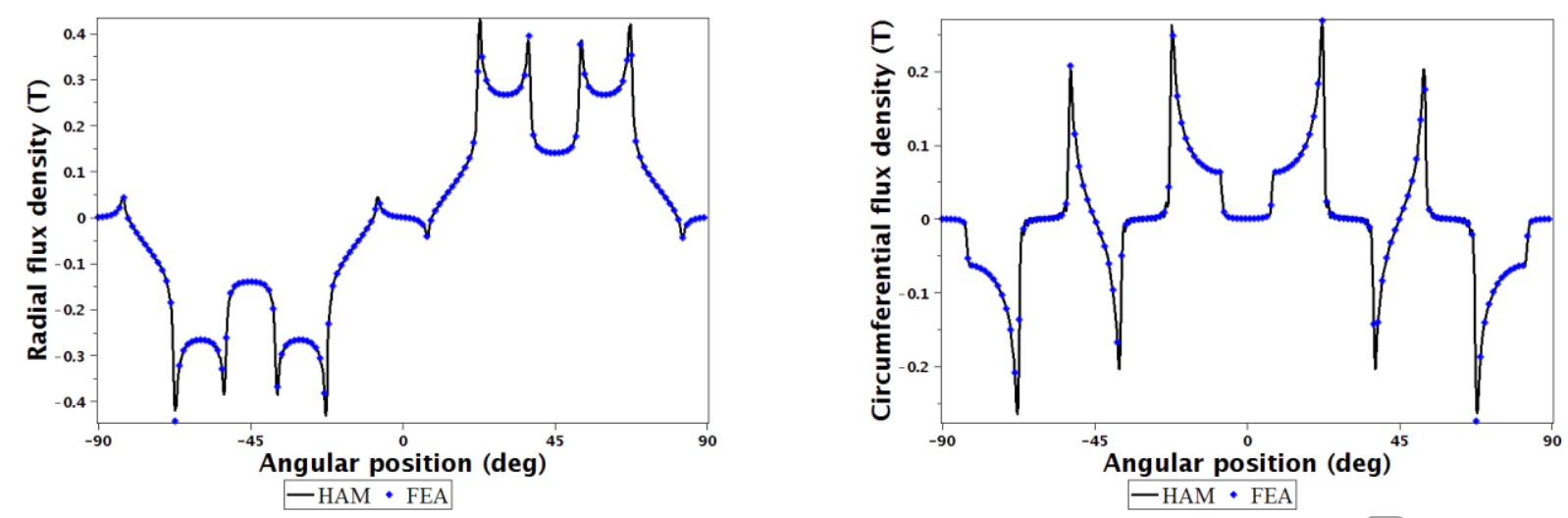

Fig. 9. Comparison of HAM and FEA predicted of the armature reaction magnetic flux density in the middle of region II for $\mu_{r}=1,000$ in the region VI.
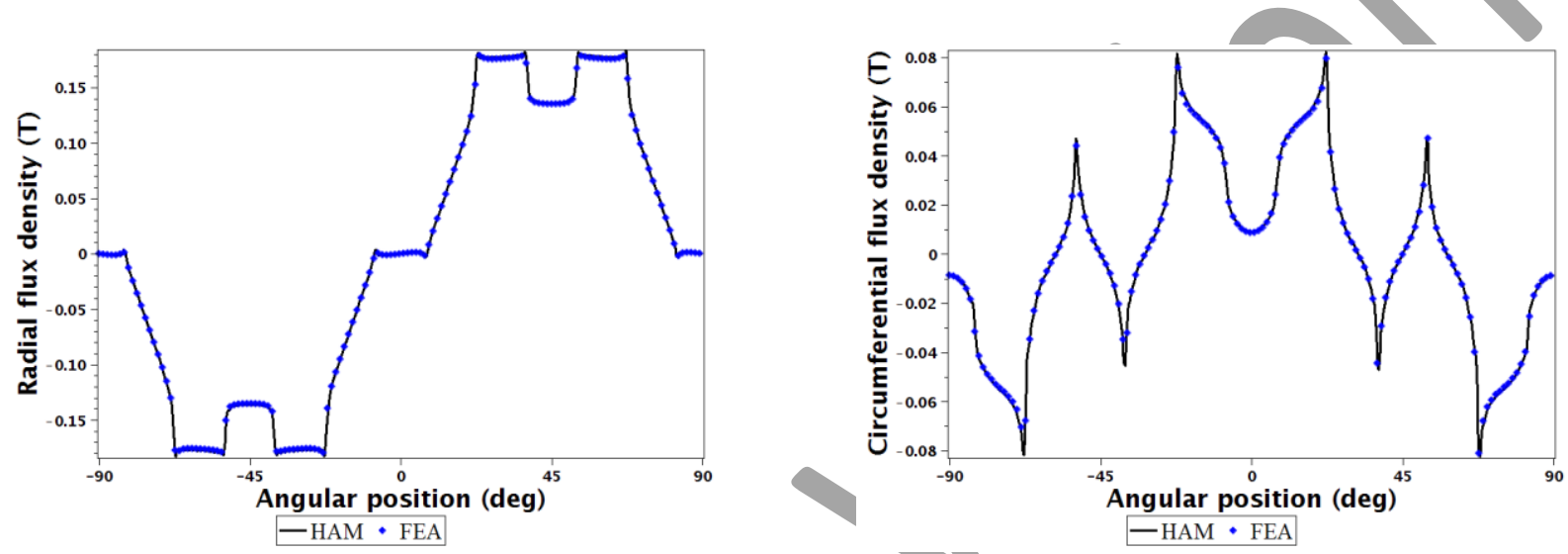

Fig. 10. Comparison of HAM and FEA predicted of the armature reaction magnetic flux density in the middle of region II for $\mu_{r}=2$ in the region VI.

Figs 11 and 12 show respectively the magnetic flux density distribution in the middle of slots and teeth under open circuit or armature reaction conditions. To avoid errors calculation, the mesh discretization in teeth regions should be selected fine $(N C=51$ and $N L=51)$ unlike to the previous case where $(N C=30$ and $N L=15$ ). Excellent agreement is achieved between HAM and FEA whatever relative permeability values. The computational time is increased and equal about 4 times.

Table II shows the time consumption for flux density calculation by a different approach, such as the HAM, SD and FEA techniques. Regarding SD technique, the HAM, proposed for this paper, in teeth regions is substituted by analytical model such indicated in (Roubache et al., 2018a) and (Ben Yahia et al., 2018a).
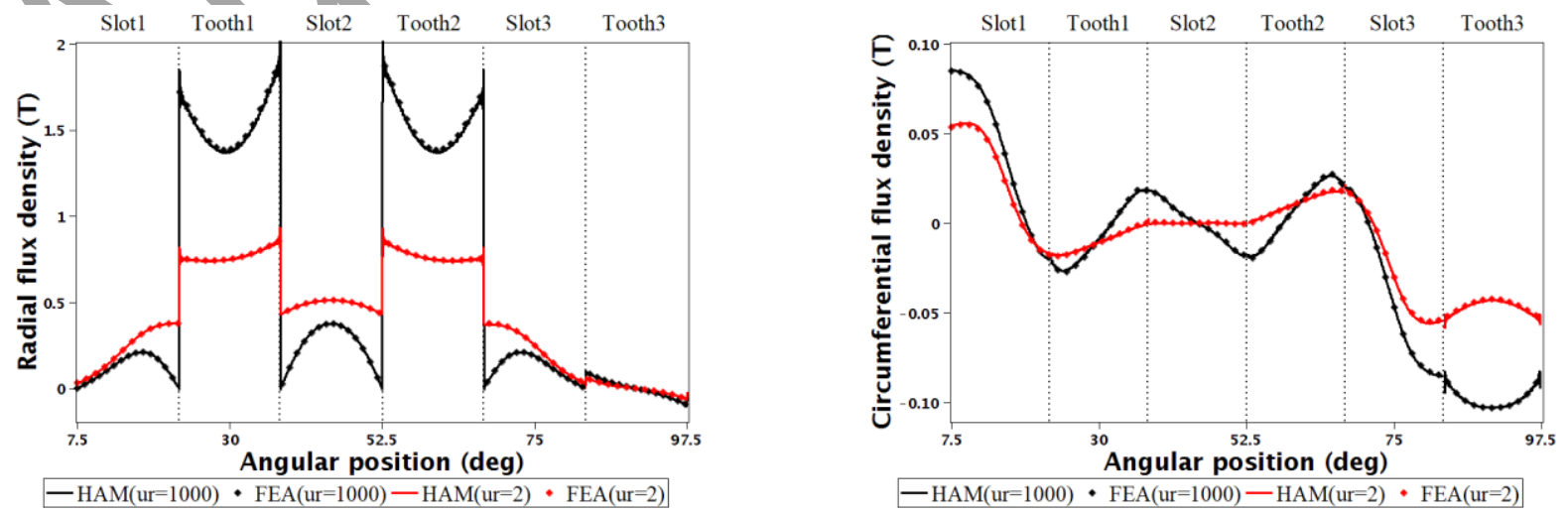

Fig. 11. Comparison of HAM and FEA predicted for the open circuit magnetic flux density distribution with a parallel magnetization pattern in the middle of region $\mathrm{V}$ and VI. 
COMPEL: The International Journal for Computation and Mathematics in Electrical and Electronic Engineering DOI: 10.1108/COMPEL-01-2021-0008
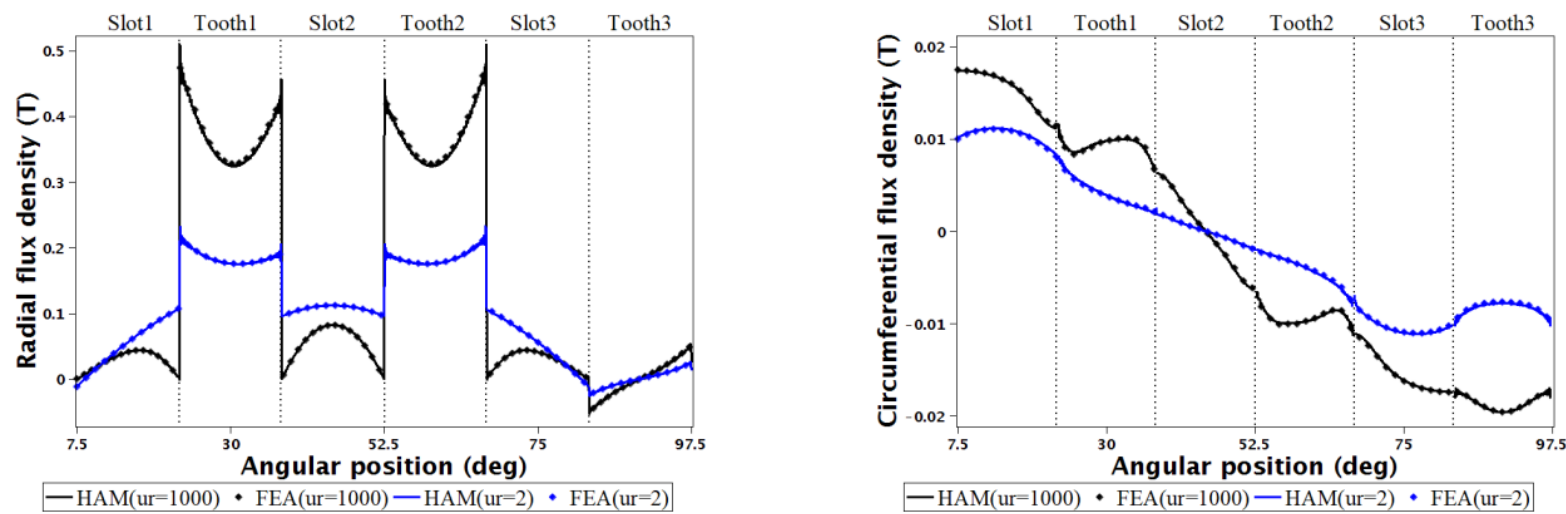

Fig. 12. Comparison of HAM and FEA predicted of the armature magnetic flux density distribution in the middle of region $\mathrm{V}$ and VI.

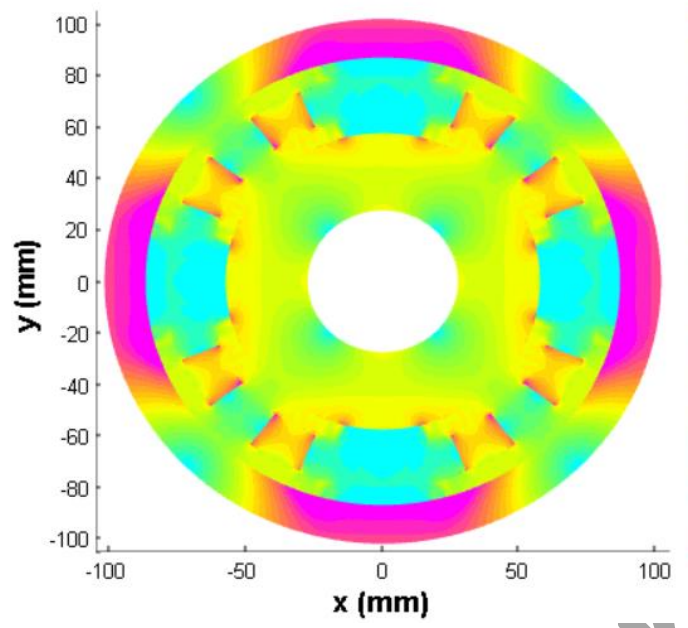

HAM
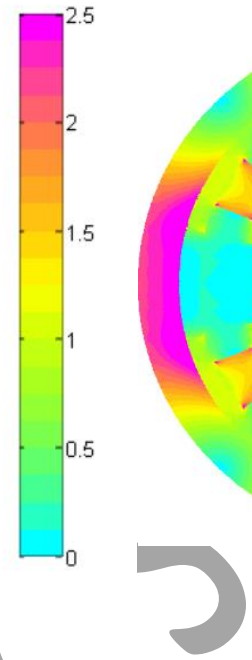

(a)
FEA
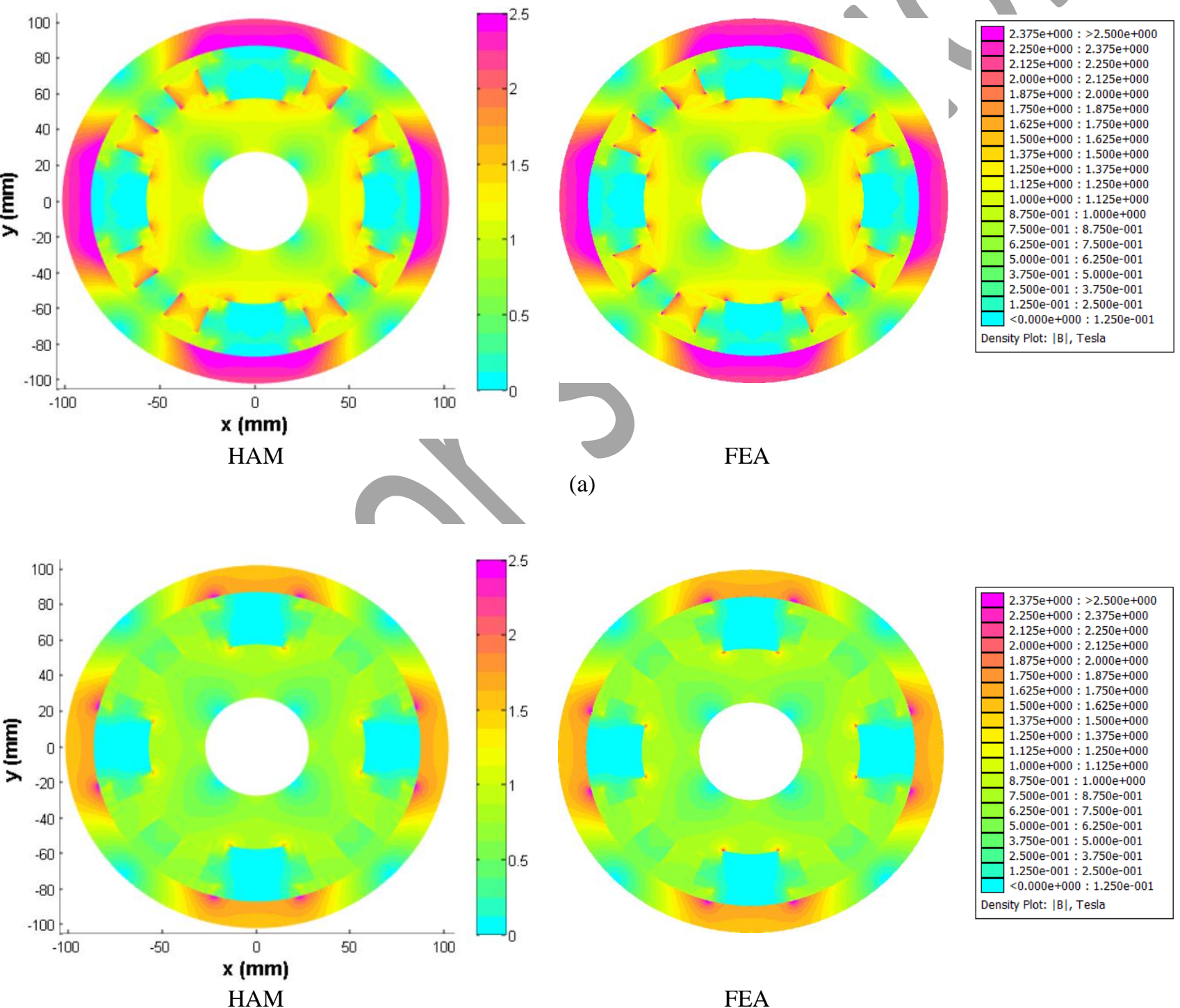

(b)

FEA

Fig. 13. Magnetic flux density $\|B\|$ distribution calculated by HAM and compared to FEA under no-load condition for: (a) $\mu_{r}=1,000$ and (b) $\mu_{r}=2$.

Figs. 13 and 14 show the magnetic flux density distribution in all parts of an electrical machine calculated by HAM and compared to FEA with different values of relative permeability of iron. The relative permeability of region VII and region VIII (see Fig. 2) are supposed equal to infinity. 

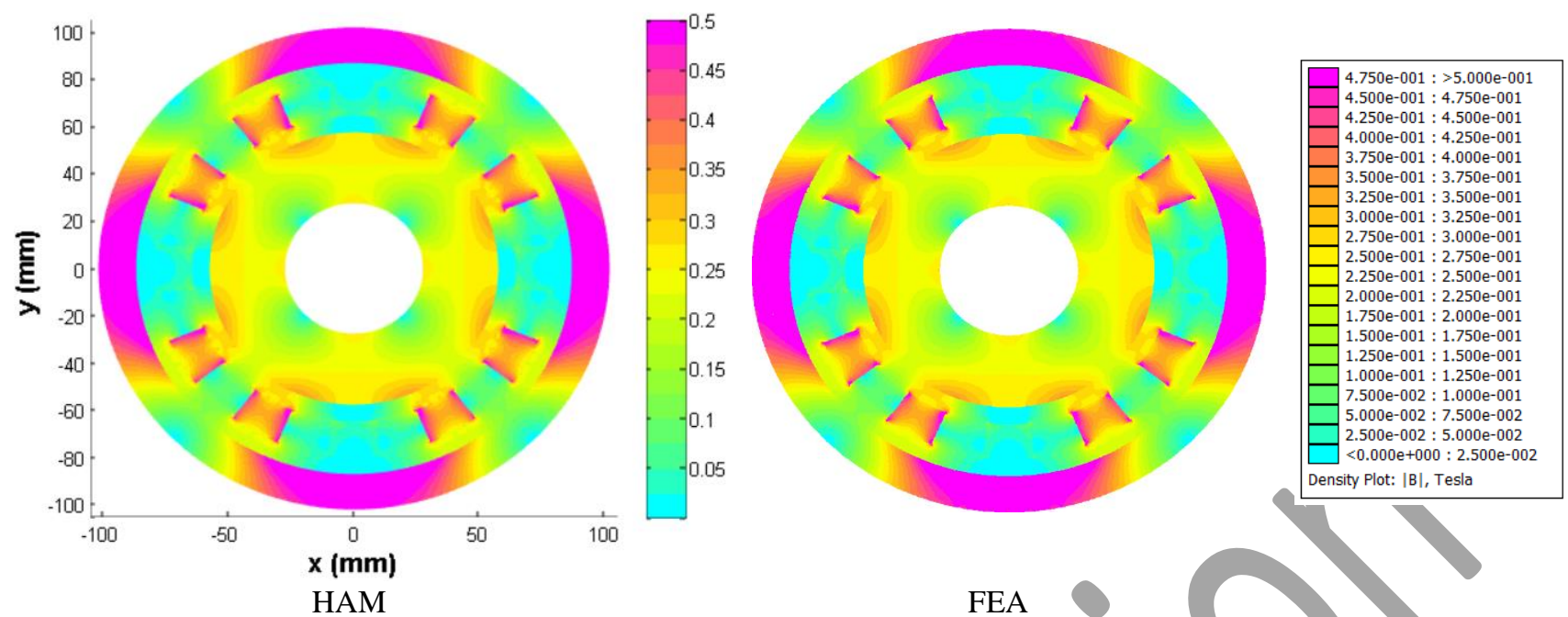

(a)
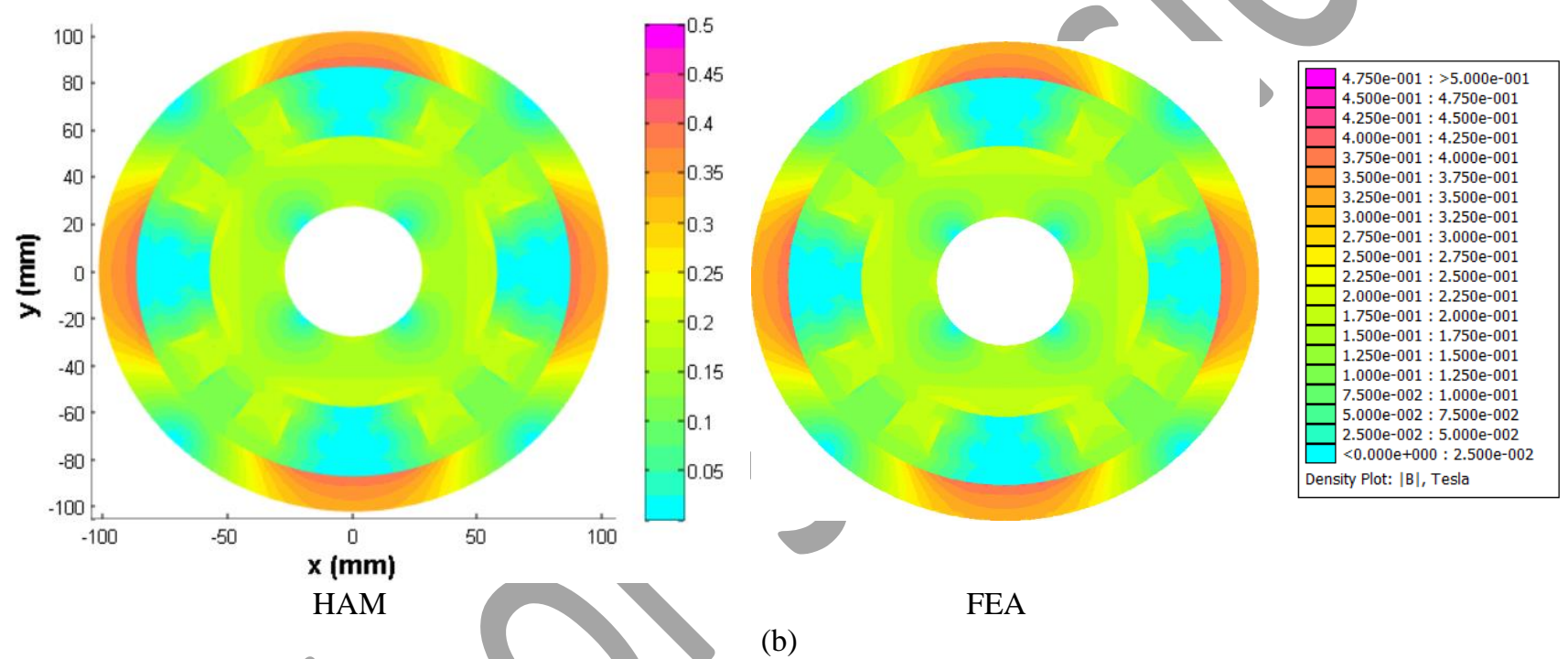

Fig. 14. Magnetic flux density $\|B\|$ distribution calculated by HAM and compared to FEA under armature reaction current condition for: (a) $\mu_{r}=1,000$ and (b) $\mu_{r}=2$.

\section{Conclusion}

In this paper, a 2-D HAM in polar coordinates has been proposed for the dual-rotor PM synchronous machines. The developed model is based on the exact AM by applying superposition technique able to be coupled to MEC in both directions (i.e., $r$ - and $\theta$-edges). The current densities in slots have been substituted by local Maxwell's equations in the slot regions to avoid using MMF situated in slots, teeth and yoke as adopted by all authors in hybrid models. Excellent results have been obtained and verified by FEA for any relative permeability value of iron core. Whatever load conditions, this approach can greatly help to optimize precisely performances machine especially with the local saturation effect which will be proposed in a future contribution.

\section{REFERENCES}

Bao, J. Gysen, B.L.J.., and Lomonova, E.A. (2018), "Hybrid analytical modeling of saturated linear and rotary electrical machines: Integration of Fourier modeling and magnetic equivalent circuits, " IEEE Trans. Magn., vol. 54, no. 11, Art. no. 8109905, DOI: 10.1109/TMAG.2018.2837896. 
Ben-Yahia, M., Boughrara, K., Dubas, F., Roubache, L., and Ibtiouen, R. (2018), “Two-dimensional exact subdomain technique of switched reluctance Machines with sinusoidal current excitation," Math. Comput. Appl., vol. 23, no. 4, p. 59, DOI: 10.3390/mca23040059.

Demenko, A., and Stachowiak, D. (2008), "Electromagnetic torque calculation using magnetic network methods," COMPEL - Int. J. Comput. Math. Electr. Electron. Eng., vol. 27, no. 1, pp. 17-26, DOI: $10.1108 / 03321640810836591$.

Ceylan, D., Friedrich, L.A. J., Boynov, K.O., and Lomonova, E.A. (2020), "Convergence analysis of the fixed-point method with the hybrid analytical modeling for 2-D nonlinear magnetostatic problems," IEEE Trans. Magn., Art. no. 7500105, DOI: 10.1109/TMAG.2020.3024539.

Djelloul-Khedda, Z., Boughrara, K., Ibtiouen, R., and Dubas, F. (2016), "Nonlinear analytical calculation of magnetic field and torque of switched reluctance machines," in Proc. CISTEM, Marrakech, Morocco, 26-28, DOI: 10.1109/CISTEM.2016.8066773.

Djelloul-Khedda, Z., Boughrara, K., Dubas, F., and Ibtiouen, R. (2017), "Nonlinear analytical prediction of magnetic field and electromagnetic performances in switched reluctance machines," IEEE Trans. Magn., vol. 53, no. 7, Art. no. 8107311, DOI: 10.1109/TMAG.2017.2679686

Djelloul-Khedda, Z., Boughrara, K., Dubas, F., Kechroud, A., and Souleyman, B. (2018), "Semianalytical magnetic field predicting in many structures of permanent-magnet synchronous machines considering the iron permeability," IEEE Trans. Magn., vol. 54, no. 7, Art. no. 8103921, DOI: 10.1109/TMAG.2018.282428.

Djelloul-Khedda, Z., Boughrara, K., Dubas, F., Kechroud, A., and Tikellaline, A. (2019), “Analytical prediction of iron-core losses in flux-modulated permanent-magnet synchronous machines," IEEE Trans. Magn., vol. 55, no. 1, Art. no. 6300112, DOI: 10.1109/TMAG.2018.2877164.

Dubas, F. and Espanet, C. (2009), "Analytical solution of the magnetic field in permanent-magnet motors taking into account slotting effect: No-load vector potential and flux density calculation," IEEE Trans. Magn., vol. 45, no. 5, pp. 2097-2109, DOI: 10.1109/TMAG.2009.2013245.

Dubas, F., and Boughrara, K. (2017a), "New scientific contribution on the 2-D subdomain technique in Cartesian coordinates: Taking into account of iron parts," Math. Comput. Appl., vol. 22, no. 1, p. 17, DOI: $10.3390 / \mathrm{mca} 22010017$.

Dubas, F., and Boughrara, K. (2017b) "New scientific contribution on the 2-D subdomain technique in polar coordinates: Taking into account of iron parts," Math. Comput. Appl., vol. 22, no. 4, p. 42, DOI:10.3390/mca22040042.

Golovanov, D., and Gerada, C. (2019a), "An analytical subdomain model for dual-rotor PM motor with Halbach array," IEEE Trans. Magn., vol. 55, no. 12, Art. no. 8205216, DOI: 10.1109/TMAG.2019.2941699.

Golovanov, D., Galassini, A., Flanagan, L., Gerada, D., Xu, Z., and Gerada, C. (2019b), "Dual-rotor permanent magnet motor for electric superbike," in Proc. IEMDC, pp. 951-956, San Diego, CA, USA, 12-15 May, 2019, DOI: 10.1109/IEMDC.2019.8785287.

Laoubi, Y., Dhifli, M., Verez, G., Amara, Y., and Barakat, G. (2015), "Open circuit performance analysis of a permanent magnet linear machine using a new hybrid analytical model," IEEE Trans. Magn., vol. 51, no. 3, Art. no. 8102304, DOI: 10.1109/TMAG.2014.2361017.

Liu, Y., Zhang, Z., Geng, W., and Li, J. (2017), “A simplified finite-element model of hybrid excitation synchronous machines with radial/axial flux paths via magnetic equivalent circuit," IEEE Trans. Magn., vol. 53, no. 11, Art. no. 7403004, DOI: 10.1109/TMAG.2017.2696568.

Meeker, D. C., (2010), "Finite Element Method Magnetics", Version 4.2, Build, http://www.femm.info. 
Nedjar, B., Vido, L., Hlioui, S., Amara, Y., and Gabsi, M. (2012), "Hybrid coupling: Magnetic equivalent circuit coupled to finite element analysis for PMSM electromagnetic modeling," in Proc. ISIE, pp. 858-862, Hangzhou, China, 28-31 May 2012, DOI: 10.1109/ISIE.2012.6237201.

Ouagued, Aden-Diriye, A., Amara, Y., and Barakat, G. (2015), "A general framework based on a hybrid analytical model for the analysis and design of permanent magnet machines," IEEE Trans. Magn., vol. 51, no. 11, Art. no. 8110204, DOI: 10.1109/TMAG.2015.2442214.

Ouagued, S., Amara, Y., and Barakat, G. (2016), "Comparison of hybrid analytical modelling and reluctance network modelling for pre-design purposes," Mathematics and Computers in Simulation, vol. 130, pp. 3-21, DOI: 10.1016/j.matcom.2016.05.001.

Philips, D.A. (1992), “Coupling finite elements and magnetic networks in magnetostatics,” Int. J. for Numerical Methods in Engineering, vol. 35, no. 10, pp. 1991-2002, DOI: 10.1002/nme.1620351005.

Ramakrishnan, K., Curti, M., Zarko, D., Mastinu, G., Paulides, J.J.H., and Lomonova, E.A. (2017), "Comparative analysis of various methods for modelling surface permanent magnet machines," IET Elect. Power Appl., vol. 11, no. 4, pp. 540-547, DOI: 10.1049/iet-epa.2016.0720.

Roubache, L., Boughrara, K., Dubas, F. and Ibtiouen, R. (2018a), "New subdomain technique for electromagnetic performances calculation in radial-flux electrical machines considering finite softmagnetic material permeability," IEEE Trans. Magn., vol. 54, no. 4, Art. no. 8103315, DOI: 10.1109/TMAG.2017.2785254.

Roubache, L., Boughrara, K., Dubas, F., and Ibtinouen, R. (2018b), “Technique en sous-domaines élémentaires dans les machines asynchrones à cage d'écureuil: Saturation magnétique locale \& Courants de Foucault dans les barres," in Proc. Symposium de Génie Électrique, Nancy, France, 3-5 Jul. 2018.

Roubache, L., Boughrara, K., Dubas, F., and Ibtiouen, R. (2019), "Elementary subdomain technique for magnetic field calculation in rotating electrical machines with local saturation effect," COMPEL Int. J. Comput. Math. Electr. Electron. Eng., vol. 38, no. 1, pp. 24-45, DOI: 10.1108/COMPEL-112017-0481.

Sprangers, R.L.J., Paulides, J.J.H., Gysen, B.L.J., Waarma, J., and Lomonova, E.A. (2015), "Semianalytical framework for synchronous reluctance motor analysis including finite soft-magnetic material permeability," IEEE Trans. Magn., vol. 51, no. 11, Art. no. 8110504, DOI: 10.1109/TMAG.2015.2442419.

Sprangers, R.L.J., Paulides, J.J.H., Gysen, B.L.J., and Lomonova, E.A. (2016), "Magnetic saturation in semi-analytical harmonic modeling for electric machine analysis," IEEE Trans. Magn., vol. 52, no. 2, Art. no. 8100410, DOI: 10.1109/TMAG.2015.2480708.

Ullah, N., Khan, F., Ullah, W., Basit, A., Umair, M., and Khattak, Z. (2018), “Analytical modelling of open-circuit flux linkage, cogging torque and electromagnetic torque for design of switched flux permanent magnet machine," Journal of Magnetics, vol.23, no. 2, pp.253-266, DOI: 10.4283/JMAG2018.23.2.253.

Wu, L., Yin, H., Wang, D., and Fang, Y. (2020), "On-load field prediction in SPM machines by a subdomain and magnetic circuit hybrid model," IEEE Trans. Ind. Elec., vol. 67, no. 9, pp. 71907201, DOI: 10.1109/TIE.2019.2942561.

Zhang, H.S., Deng, Z.X., Yang, M.L., Zhang, Y., Tuo, J.Y., and Xu, J. (2020), “Analytical prediction of Halbach array permanent magnet machines considering finite tooth permeability," IEEE Trans. Magn., vol. 56, no. 6, Art. no. 8101010, DOI: 10.1109/TMAG.2020.2982844. 


\section{Appendix A}

Parallel magnetization expressions in (5) are defined by:

- for the radial component:

$$
\begin{aligned}
& M r_{r s n}=m_{r n} \cdot \frac{B_{r m}}{\mu_{o}} \cdot \sin \left(\frac{n \pi}{2}\right) \cdot \cos (n p \cdot \tau) \\
& M r_{r c n}=m_{r n} \cdot \frac{B_{r m}}{\mu_{o}} \cdot \sin \left(\frac{n \pi}{2}\right) \cdot \sin (n p \cdot \tau)
\end{aligned}
$$

- for the circumferential component:

$$
\begin{aligned}
& M r_{\theta s n}=-m_{\theta n} \cdot \frac{B_{r m}}{\mu_{o}} \cdot \sin \left(\frac{n \pi}{2}\right) \cdot \sin (n p \cdot \tau) \\
& M r_{\theta c n}=m_{\theta n} \cdot \frac{B_{r m}}{\mu_{o}} \cdot \sin \left(\frac{n \pi}{2}\right) \cdot \cos (n p \cdot \tau)
\end{aligned}
$$

where $B_{r m}$ is the remanent flux density of PMs, $\tau$ is the angular position of PMs, and

$$
m_{r n}=\frac{4}{\pi} \cdot \int_{0}^{\frac{\beta \pi}{2}} \cos \left(\frac{\theta}{p}\right) \cdot \cos (n \theta) \cdot d \theta=\left\{\begin{array}{l}
\frac{p}{\pi} \cdot \sin \left(\frac{\beta \pi}{p}\right)+\beta \\
\text { if } n p=1 \\
\frac{2 p}{\pi} \cdot\left\{\frac{\sin \left[(n p-1) \cdot \frac{\beta \pi}{2 p}\right]}{(n p-1)}+\frac{\sin \left[(n p+1) \cdot \frac{\beta \pi}{2 p}\right]}{(n p+1)}\right\} \text { if } n p \neq 1
\end{array}\right.
$$

and

$$
m_{\theta n}=\frac{4}{\pi} \cdot \int_{0}^{\frac{\beta \pi}{2}} \sin \left(\frac{\theta}{p}\right) \cdot \sin (n \theta) \cdot d \theta= \begin{cases}-\frac{p}{\pi} \cdot \sin \left(\frac{\beta \pi}{p}\right)+\beta & \text { if } n p=1 \\ \frac{2 p}{\pi} \cdot\left\{\frac{\sin \left[(n p-1) \cdot \frac{\beta \pi}{2 p}\right]}{(n p-1)}-\frac{\sin \left[(n p+1) \cdot \frac{\beta \pi}{2 p}\right]}{(n p+1)}\right\} \text { if } n p \neq 1\end{cases}
$$

with $\beta$ is the PM pole-arc to pole-pitch ratio.

\section{APPENDIX B}

From (20), we have:

$$
\begin{aligned}
& n p \cdot\left(C_{3 n}^{I} \cdot R_{1}^{n p-1}-C_{4 n}^{I} \cdot R_{1}^{-n p-1}\right)=-\left.\frac{d \Gamma_{s}}{d r}\right|_{r=R_{1}}+\frac{1}{\mu_{0}} \cdot M r_{\theta s n} \\
& n p \cdot\left(C_{5 n}^{I} \cdot R_{1}^{n p-1}-C_{6 n}^{I} \cdot R_{1}^{-n p-1}\right)=-\left.\frac{d \Gamma_{c}}{d r}\right|_{r=R_{1}}+\frac{1}{\mu_{0}} \cdot M r_{\theta c n}
\end{aligned}
$$

From (21), we have:

$$
\begin{aligned}
& C_{3 n}^{I} \cdot R_{2}^{n p}+C_{4 n}^{I} \cdot R_{2}^{-n p}-C_{3 n}^{I I} \cdot R_{2}^{n p}-C_{4 n}^{I I} \cdot R_{2}^{-n p}=-\left.\Gamma_{s}\right|_{r=R_{2}} \\
& C_{5 n}^{I} \cdot R_{2}^{n p}+C_{6 n}^{I} \cdot R_{2}^{-n p}-C_{5 n}^{I I} \cdot R_{2}^{n p}-C_{6 n}^{I I} \cdot R_{2}^{-n p}=-\left.\Gamma_{c}\right|_{r=R_{2}}
\end{aligned}
$$


From (22), we have:

$$
\begin{aligned}
& n p \cdot\left(C_{3 n}^{I} \cdot R_{2}^{n p-1}-C_{4 n}^{I} \cdot R_{2}^{-n p-1}-C_{3 n}^{I I} \cdot R_{2}^{n p-1}+C_{4 n}^{I I} \cdot R_{2}^{-n p-1}\right)=-\left.\frac{d \Gamma_{s}}{d r}\right|_{r=R_{2}}+\frac{1}{\mu_{0}} \cdot M r_{\theta s n} \\
& n p \cdot\left(C_{5 n}^{I} \cdot R_{2}^{n p-1}-C_{6 n}^{I} \cdot R_{2}^{-n p-1}-C_{5 n}^{I I} \cdot R_{2}^{n p-1}+C_{6 n}^{I I} \cdot R_{2}^{-n p-1}\right)=-\left.\frac{d \Gamma_{c}}{d r}\right|_{r=R_{2}}+\frac{1}{\mu_{0}} \cdot M r_{\theta c n}
\end{aligned}
$$

Development of (24) gives:

$$
\begin{gathered}
C_{x 1}^{V}+C_{x 2}^{V} \cdot \ln \left(R_{3}\right)-\frac{1}{4} \mu_{o} \cdot J_{z x} \cdot R_{3}^{2}=\frac{1}{w} \cdot \int_{g_{x}-\frac{w}{2}}^{g_{x}+\frac{w}{2}} A_{z}^{I I}\left(R_{3}, \theta\right) \cdot d \theta \\
C_{x 3 m}^{V} \cdot\left(\frac{R_{3}}{R_{4}}\right)^{\beta_{m}}+C_{x 4 m}^{V}=\frac{2}{w} \cdot \int_{g_{x}-\frac{w}{2}}^{g_{x}+\frac{w}{2}} A_{z}^{I I}\left(R_{3}, \theta\right) \cdot \cos \left[\beta_{m} \cdot\left(\theta-g_{x}+\frac{w}{2}\right)\right] \cdot d \theta
\end{gathered}
$$

From (25), we have:

$$
\begin{aligned}
&-\mu_{0} n p \cdot\left(C_{3 n}^{I I} \cdot R_{3}^{n p-1}-C_{4 n}^{I I} \cdot R_{3}^{-n p-1}\right)= \frac{1}{\pi} \cdot \sum_{x=1}^{Q} \int_{g_{x}-\frac{w}{2}}^{g_{x}+\frac{w}{2}} H_{\theta x}^{V}\left(R_{3}, \theta\right) \cdot \sin (n p \theta) \cdot d \theta+\ldots \\
& \frac{1}{\pi} \cdot \sum_{x=1}^{Q} \int_{g_{x}+\frac{w}{2}}^{g_{x+1}-\frac{w}{2}} H_{\theta x}^{V I}\left(R_{3}, \theta\right) \cdot \sin (n p \theta) \cdot d \theta \\
&-\mu_{0} n p \cdot\left(C_{5 n}^{I I} \cdot R_{3}^{n p-1}-C_{6 n}^{I I} \cdot R_{3}^{-n p-1}\right)= \frac{1}{\pi} \cdot \sum_{x=1}^{Q} \int_{g_{x}-\frac{w}{2}}^{g_{x}+\frac{w}{2}} H_{\theta x}^{V}\left(R_{3}, \theta\right) \cdot \cos (n p \theta) \cdot d \theta+\ldots \\
& \frac{1}{\pi} \cdot \sum_{x=1}^{Q} \int_{g_{x+1}-\frac{w}{2}} H_{\theta x}^{V I}\left(R_{3}, \theta\right) \cdot \cos (n p \theta) \cdot d \theta
\end{aligned}
$$

where the Fourier constants of (26) can be written as:

$$
\begin{aligned}
& h_{\theta x s v}^{V I}=\frac{2 p}{\pi} \cdot \int_{\theta_{x, j}}^{\theta_{x, j}+\Delta \theta} \sin (v p \theta) \cdot d \theta \\
& h_{\theta x c v}^{V I}=\frac{2 p}{\pi} \cdot \int_{\theta_{x, j}}^{\theta_{x, j}+\Delta \theta} \cos (v p \theta) \cdot d \theta
\end{aligned}
$$

Development of (28) gives: 
COMPEL: The International Journal for Computation and Mathematics in Electrical and Electronic Engineering DOI: 10.1108/COMPEL-01-2021-0008

$$
\begin{gathered}
C_{x 1}^{V}+C_{x 2}^{V} \cdot \ln \left(R_{4}\right)-\frac{1}{4} \mu_{o} \cdot J_{z x} \cdot R_{4}^{2}=\frac{1}{w} \cdot \int_{g_{x}-\frac{w}{2}}^{g_{x}+\frac{w}{2}} A_{z}^{I I I}\left(R_{3}, \theta\right) \cdot d \theta \\
C_{x 3 m}^{V}+C_{x 4 m}^{V} \cdot\left(\frac{R_{4}}{R_{3}}\right)^{-\beta_{m}}=\frac{2}{w} \int_{g_{x}-\frac{w}{2}}^{g_{x}+\frac{w}{2}} A_{z}^{I I I}\left(R_{3}, \theta\right) \cdot \cos \left[\beta_{m} \cdot\left(\theta-g_{x}+\frac{w}{2}\right)\right] \cdot d \theta
\end{gathered}
$$

From (29), we have:

$$
\begin{aligned}
&-\mu_{0} n p \cdot\left(C_{3 n}^{I I I} \cdot R_{4}^{n p-1}-C_{4 n}^{I I I} \cdot R_{4}^{-n p-1}\right)= \frac{1}{\pi} \cdot \sum_{x=1}^{Q} \int_{g_{x}-\frac{w}{2}}^{g_{x}+\frac{w}{2}} H_{\theta x}^{V}\left(R_{4}, \theta\right) \cdot \sin (n p \theta) \cdot d \theta+\cdots \\
&-\mu_{0} n p \cdot\left(C_{5 n}^{I I I} \cdot R_{4}^{n p-1}-C_{6 n}^{I I I} \cdot R_{4}^{-n p-1}\right)= \frac{1}{\pi} \cdot \sum_{x=1}^{g_{x+1}-\frac{w}{2}} \int_{g_{x}+\frac{w}{2}} H_{\theta x}^{V I}\left(R_{4}, \theta\right) \cdot \sin (n p \theta) \cdot d \theta \\
& \frac{1}{\pi} \cdot \sum_{x=1}^{Q} \int_{g_{x}+\frac{w}{2}} H_{\theta x}^{V}\left(R_{4}, \theta\right) \cdot \cos (n p \theta) \cdot d \theta+\cdots \\
& \frac{1}{\pi} \sum_{x=1}^{g_{x+1}} \int_{g_{x}+\frac{w}{2}} H_{\theta x}^{V I}\left(R_{4}, \theta\right) \cdot \cos (n p \theta) \cdot d \theta
\end{aligned}
$$

From (30), we have:

$$
\begin{aligned}
& C_{3 n}^{I V} \cdot R_{5}^{n p}+C_{4 n}^{I V} \cdot R_{5}^{-n p}-C_{3 n}^{I I I} \cdot R_{5}^{n p}-C_{4 n}^{I I I} \cdot R_{5}^{-n p}=-\left.\Gamma_{s}\right|_{r=R_{5}} \\
& C_{5 n}^{I V} \cdot R_{5}^{n p}+C_{6 n}^{I V} \cdot R_{5}^{-n p}-C_{5 n}^{I I I} \cdot R_{5}^{n p}-C_{6 n}^{I I I} \cdot R_{5}^{-n p}=-\left.\Gamma_{c}\right|_{r=R_{5}}
\end{aligned}
$$

From (31), we have:

$$
\begin{aligned}
& n p \cdot\left(C_{3 n}^{I V} \cdot R_{5}^{n p-1}-C_{4 n}^{I V} \cdot R_{5}^{-n p-1}-C_{3 n}^{I I I} \cdot R_{5}^{n p-1}+C_{4 n}^{I I I} \cdot R_{5}^{-n p-1}\right)=-\left.\frac{d \Gamma_{s}}{d r}\right|_{r=R_{5}}+\frac{1}{\mu_{0}} \cdot M r_{\theta s n} \\
& n p \cdot\left(C_{5 n}^{I V} \cdot R_{5}^{n p-1}-C_{6 n}^{I V} \cdot R_{5}^{-n p-1}-C_{5 n}^{I I I} \cdot R_{5}^{n p-1}+C_{6 n}^{I I I} \cdot R_{5}^{-n p-1}\right)=-\left.\frac{d \Gamma_{c}}{d r}\right|_{r=R_{5}}+\frac{1}{\mu_{0}} \cdot M r_{\theta c n}
\end{aligned}
$$

From (32), we have:

$$
\begin{aligned}
& n p \cdot\left(C_{3 n}^{I V} \cdot R_{6}^{n p-1}-C_{4 n}^{I V} \cdot R_{6}^{-n p-1}\right)=-\left.\frac{d \Gamma_{s}}{d r}\right|_{r=R_{6}}+\frac{1}{\mu_{0}} \cdot M r_{\theta s n} \\
& n p \cdot\left(C_{5 n}^{I V} \cdot R_{6}^{n p-1}-C_{6 n}^{I V} \cdot R_{6}^{-n p-1}\right)=-\left.\frac{d \Gamma_{c}}{d r}\right|_{r=R_{6}}+\frac{1}{\mu_{0}} \cdot M r_{\theta c n}
\end{aligned}
$$

From (34) and (35), we have:

$$
\frac{\lambda_{v} \cdot\left(C_{x 5 v}^{V} \cdot \cosh \left(\lambda_{v} \cdot w\right)+C_{x 6 v}^{V}\right)}{\sinh \left(\lambda_{v} \cdot w\right)}=-\sum_{a=1}^{N L-1} r_{a+1} \cdot \mu_{0} \cdot\left(\frac{u_{x, a+1}-u_{x, a}}{\Delta r}\right) \cdot h_{r x v}^{V I}
$$


where

$$
h_{r x v}^{V I}=\frac{2}{\ln \left(\frac{R_{4}}{R_{3}}\right)} \cdot \int_{r_{k}}^{r_{k}+\Delta R} \frac{1}{r} \cdot \sin \left[\lambda_{v} \cdot \ln \left(\frac{r}{R_{3}}\right)\right] \cdot d r
$$

From (37), we have:

$$
\frac{\lambda_{v} \cdot\left(C_{x 6 v}^{V} \cdot \cosh \left(\lambda_{v} \cdot w\right)+C_{x 5 v}^{V}\right)}{\sinh \left(\lambda_{v} \cdot w\right)}=-\sum_{b=1}^{N L-1} r_{b+1} \cdot \mu_{0} \cdot\left(\frac{u_{x, b+1}-u_{x, b}}{\Delta r}\right) \cdot h_{r x v}^{V I}
$$

Some integral functions are defined as:

- $1^{\text {st }}$ integral:

$$
\int_{g_{x}-\frac{w}{2}}^{g_{x}+\frac{w}{2}} \sin (n p \theta) \cdot d \theta=\frac{2 \sin \left(n p \cdot \frac{w}{2}\right) \cdot \sin \left(n p \cdot g_{x}\right)}{n p}
$$

- $2^{\text {nd }}$ integral:

$$
\int_{g_{x}-\frac{w}{2}}^{g_{x}+\frac{w}{2}} \cos (n p \theta) \cdot d \theta=\frac{2 \sin \left(n p \cdot \frac{w}{2}\right) \cdot \cos \left(n p \cdot g_{x}\right)}{n p}
$$

- $3^{\text {rd integral: }}$

$$
\begin{aligned}
& g_{x}+\frac{w}{2} \\
& \int_{g_{x}-\frac{w}{2}} \sin (n p \theta) \cdot \cos \left[\beta_{m} \cdot\left(\theta-g_{x}+\frac{w}{2}\right)\right] \cdot d \theta= \\
& \begin{cases}\frac{(n p)^{2}}{\left(\beta_{m}\right)^{2}-(n p)^{2}} \cdot\left\{\cos \left[n p \cdot\left(g_{x}+\frac{w}{2}\right)\right](-1)^{m}-\cos \left[n p \cdot\left(g_{x}-\frac{w}{2}\right)\right]\right\} & \text { if } \beta_{m} \neq n p \\
\frac{m \pi}{2 n p} \cdot \cos \left(n p g_{x}-(m+1) \cdot \frac{\pi}{2}\right) & \text { if } \beta_{m}=n p\end{cases}
\end{aligned}
$$

- 4th integral:

$$
\begin{gathered}
\int_{g_{x}-\frac{w}{2}}^{g_{x}+\frac{w}{2}} \cos (n p \theta) \cdot \cos \left[\beta_{m} \cdot\left(\theta-g_{x}+\frac{w}{2}\right)\right] d \theta= \\
\begin{cases}\frac{(n p)^{2}}{\left(\beta_{m}\right)^{2}-(n p)^{2}} \cdot\left\{\sin \left[n p \cdot\left(g_{x}+\frac{w}{2}\right)\right](-1)^{m}-\sin \left[n p\left(g_{x}-\frac{w}{2}\right)\right]\right\} & \text { if } \beta_{m} \neq n p(B 29 a) \\
\frac{m \pi}{2 n p} \cdot \cos \left(n p g_{x}-m \cdot \frac{\pi}{2}\right) & \text { if } \beta_{m}=n p(B 29 b)\end{cases}
\end{gathered}
$$

- 5th integral: 
COMPEL: The International Journal for Computation and Mathematics in Electrical and Electronic Engineering DOI: 10.1108/COMPEL-01-2021-0008

$$
\begin{gathered}
\int_{g_{x}-\frac{w}{2}}^{g_{x}+\frac{w}{2}} \sin (n p \theta) \cdot \sin (n p \theta) \cdot d \theta= \\
\frac{1}{4 n p} \cdot\left\{2 n p w-\sin \left[2 n p \cdot\left(g_{x}+\frac{w}{2}\right)\right]+\sin \left[2 n p \cdot\left(g_{x}-\frac{w}{2}\right)\right]\right\}
\end{gathered}
$$

- 6th integral:

$$
\begin{gathered}
\int_{g_{x}+\frac{w}{2}}^{g_{2}} \cos (n p \theta) \cdot \cos (n p \theta) \cdot d \theta= \\
\frac{1}{4 n p} \cdot\left\{2 n p w+\sin \left[2 n p \cdot\left(g_{x}+\frac{w}{2}\right)\right]-\sin \left[2 n p \cdot\left(g_{x}-\frac{w}{2}\right)\right]\right\}
\end{gathered}
$$

